\title{
Parameters Influencing Interfacial Morphology in GaAs/Ge Superlattices Grown by Metal Organic Chemical Vapor Deposition
}

\author{
Roger Jia ${ }^{1}$ and Eugene A. Fitzgerald \\ Department of Materials Science and Engineering, Massachusetts Institute of Technology, \\ Cambridge, MA 02139, USA \\ ${ }^{1}$ Corresponding Author: Phone: 248-885-5586, Email: jiaroger@mit.edu \\ Keywords: A1. Interfaces; A3. Metalorganic chemical vapor deposition; B2. Semiconducting \\ gallium arsenide; B2. Semiconducting germanium; B2. Semiconducting III-V materials
}

\begin{abstract}
We investigated the epitaxy and morphology of GaAs/Ge superlattices grown by metal organic chemical vapor deposition (MOCVD) under a range of conditions. The surfaces of Ge layers deposited on GaAs at $650^{\circ} \mathrm{C}$ and 100 Torr are observed to be rough in cross-sectional transmission electron microscopy. When either the temperature is lowered to $500^{\circ} \mathrm{C}$ or the pressure is increased to 250 Torr, the surface of the first deposited Ge layer is observed to be smooth. This behavior suggests that Ge roughening is a thermodynamically favorable process that can be kinetically limited with appropriate growth conditions. At $500^{\circ} \mathrm{C}$, GaAs islands on Ge do not completely coalesce into one film. This may result from poor surface coverage; the short depositions would not be sufficient to coarsen and completely coalesce the islands. At $650^{\circ} \mathrm{C}$, growth on offcut substrates did not suppress antiphase boundaries, likely due to the unique conditions for GaAs/Ge superlattice growth. A wide-range of two- and threedimensional nanostructures are formed and should allow insight in structure-property correlations in semiconducting thermoelectric materials.
\end{abstract}




\section{Introduction}

The efficiency of a thermoelectric is governed by the figure of merit $z T=\frac{\sigma S^{2}}{\kappa_{e}+\kappa_{l}} T$ where $\sigma$ is the electrical conductivity, $S$ is the Seebeck coefficient, $\kappa_{e}$ is the electronic thermal conductivity, and $\kappa_{l}$ is the lattice thermal conductivity. Large improvements in the figure of merit have been experimentally reported in nanostructured materials, such as superlattices, over their corresponding bulk materials [1-3], yet scale-up to representatively efficient devices has been elusive. Models have shown that the higher efficiencies from nanostructured materials should be due primarily to the reduction in $\kappa_{l}$ as a result of increased disruption to phonon transport from interfaces $[4,5]$.

Conventional semiconductors, such as silicon, germanium, GaAs, and InP, typically have good electrical conductivities and Seebeck coefficients, but they also have very high thermal conductivities, the primary reason for their poor performance as thermoelectric materials. This, however, makes superlattice structures composed of these conventional semiconductors attractive material systems to investigate. Silicon-germanium superlattices [6] and AlGaAs superlattices [7] have been studied previously for their potential as thermoelectrics. The fabrication of these materials has been well established, but their thermoelectric efficiencies are much lower than that of the more commonly used $\mathrm{Bi}_{2} \mathrm{Te}_{3}$ based materials.

Superlattice systems consisting of a III-V compound alloy, such as InGaAsP, and a SiGe alloy can in principle produce much more disruption to phonon transport than either a silicongermanium superlattice or an AlGaAs superlattice and lead to better thermoelectric efficiencies. However, fabrication of these complex superlattices has never been demonstrated due to the many challenges involved in controlling the epitaxy. The GaAs/Ge system is the simplest III- 
V/IV system with very little lattice mismatch; thus it is an ideal system to investigate the

production of controlled nanostructures. A few groups have grown $\mathrm{GaAs} / \mathrm{Ge}$ superlattices using molecular beam epitaxy (MBE) previously [8-10]; however, GaAs/Ge superlattices grown by metal organic chemical vapor deposition (MOCVD) have not been demonstrated. MOCVD is a more suitable technique for high volume fabrication, but the chemistry involved in epitaxy is more complicated. In this work, we investigated the growth and morphology of GaAs/Ge superlattices grown by MOCVD under a wide range of conditions to understand their effects and serve as a basis for growth of more complex superlattices.

\section{Experiment}

Epitaxy of GaAs/Ge superlattices was performed in a Thomas Swan/Aixtron close-coupled showerhead MOCVD reactor. Films were grown on exact (100) GaAs substrates and (100) GaAs substrates offcut $6^{\circ}$ toward the $<111>\mathrm{A}$ direction. Trimethylgallium (TMGa), $\mathrm{AsH}_{3}$, and $\mathrm{GeH}_{4}$ were the precursors used, and nitrogen was the carrier gas. A thin GaAs homoepitaxial layer was grown on all substrates to ensure a high quality surface for film growth. Samples were grown at $500^{\circ} \mathrm{C}$ or $650^{\circ} \mathrm{C}$ and at reactor pressures of 100 Torr or 250 Torr (Table 1). These temperatures were chosen both because of practical growth rates as well as previous knowledge of obtaining desired GaAs/Ge interfaces. All GaAs layers were grown at a V/III ratio above 100 .

Bai et al. found that high quality Ge on GaAs could be obtained by preparing a Ga-rich surface prior to deposition [11]. This was done either through additional TMGa flow or by annealing in $\mathrm{N}_{2}$ ambient for 10 minutes prior to $\mathrm{GeH}_{4}$ flow. Samples $1-6$ and $11-14$ were prepared in a manner similar to that used by Bai et al. Upon completion of the GaAs layer, $\mathrm{AsH}_{3}$ flow was 
shut off for 10 seconds. This was done to remove any precursors in the ambient. After 10 seconds, TMGa was flown into the reactor chamber; details of the TMGa "pulse" are shown in Table 1. After TMGa flow was stopped an additional 10 seconds were allotted to purge the ambient, followed by Ge deposition. For samples 7 - 10, no TMGa pulse was done; instead only the 20 second of anneal in $\mathrm{N}_{2}$ ambient was kept.

In the following sections, all samples mentioned will be followed by the conditions used (temperature/pressure/"Ga pulse" or "anneal only"/substrate orientation) for ease of reading.

In-situ monitoring of the reflectivity of the growing surface was done using EpiTT at a $635 \mathrm{~nm}$ wavelength. The information obtained can be used to observe growth transitions and incubation times. Layer thicknesses and morphology of the samples was analyzed using cross-sectional transmission electron microscopy (XTEM); images were obtained using a JOEL 2011 microscope. TEM samples were prepared by mechanical polishing, followed by Ar ion milling in a Fischione 1010 ion mill.

\section{Results}

\subsection{High Temperature Growth}

XTEM images of sample $1\left(650^{\circ} \mathrm{C} / 100 \mathrm{Torr} / \mathrm{Ga}\right.$ pulse/exact $)$ and sample $2\left(650^{\circ} \mathrm{C} / 100 \mathrm{Torr} / \mathrm{Ga}\right.$ pulse $/ 6^{\circ}$ off) are shown in Figure 1. The presence of antiphase boundaries (APBs) in both samples was confirmed from observing contrast reversal between (002) darkfield and (00-2) darkfield images. 
Images of sample $3\left(650^{\circ} \mathrm{C} / 250 \mathrm{Torr} / \mathrm{Ga}\right.$ pulse/exact $)$, sample $5\left(650^{\circ} \mathrm{C} / 250 \mathrm{Torr} / \mathrm{anneal}\right.$ only/exact $)$, sample $4\left(650^{\circ} \mathrm{C} / 250 \mathrm{Torr} / \mathrm{Ga}\right.$ pulse $\left./ 6^{\circ} \mathrm{off}\right)$, and sample $6\left(650^{\circ} \mathrm{C} / 250 \mathrm{Torr} /\right.$ anneal only $/ 6^{\circ}$ off) are shown in Figure 2. The samples show that the use of a TMGa pulse results in Ge layers that are much less uniform as compared to samples using just the $\mathrm{N}_{2}$ anneal; the $\mathrm{Ge}$ growth rate for samples using the TMGa pulse appears to be greatly reduced in localized regions. In addition, a wave-like characteristic can be observed in the both $6^{\circ}$ offcut samples that is less pronounced in the exact samples.

Superlattice samples with smaller period thicknesses were also grown; images of the samples are shown in Figure 3. Localized reduction in growth rate is observed for both Ge and GaAs in both samples $7\left(650^{\circ} \mathrm{C} / 250 \mathrm{Torr} / \mathrm{Ga}\right.$ pulse/exact $)$ and $8\left(650^{\circ} \mathrm{C} / 250 \mathrm{Torr} / \mathrm{Ga}\right.$ pulse $\left./ 6^{\circ} \mathrm{off}\right)$, resulting in "well" formations, extending through multiple layers. In sample $10\left(650^{\circ} \mathrm{C} / 250 \mathrm{Torr} / \mathrm{anneal}\right.$ only $/ 6^{\circ}$ off), growth of GaAs appears inhibited in localized areas.

\subsection{Low Temperature Growth}

Images of sample $11\left(500^{\circ} \mathrm{C} / 100 \mathrm{Torr} / \mathrm{Ga}\right.$ pulse/exact $)$ and sample $12\left(500^{\circ} \mathrm{C} / 100 \mathrm{Torr} / \mathrm{Ga}\right.$ pulse $/ 6^{\circ}$ off) are shown in Figure 4 . Unlike the samples grown at $650^{\circ} \mathrm{C}$, these lower temperature growths had approximately a 100 second incubation time during the deposition of the first Ge layer. Substrate orientation also affected the growth rate of the first Ge layer; the growth rate of 
Ge after incubation was $0.5 \mathrm{~nm} / \mathrm{s}$ when using an exact substrate but only $0.1 \mathrm{~nm} / \mathrm{s}$ when using a $6^{\circ}$ offcut substrate. For both samples the $\mathrm{GeH}_{4}$ flow was $629 \mu \mathrm{mol} / \mathrm{min}$.

Subsequent deposited Ge layers had very low growth rates. The incubation times for these $\mathrm{Ge}$ layers were difficult to determine from the reflectivity results due to the slow growth rate and non-uniformity effects from the growing surface.

Samples $13\left(500^{\circ} \mathrm{C} / 250 \mathrm{Torr} / \mathrm{Ga}\right.$ pulse/exact $)$ and $14\left(500^{\circ} \mathrm{C} / 250 \mathrm{Torr} / \mathrm{Ga}\right.$ pulse $/ 6^{\circ}$ off $)$ are shown in Figure 5. Incubation time for the first Ge layer was approximately 50 seconds. Growth rate was again affected by substrate orientation; the growth rate of Ge after incubation was $1 \mathrm{~nm} / \mathrm{s}$ for the exact sample and $0.4 \mathrm{~nm} / \mathrm{s}$ for the $6^{\circ}$ offcut sample. The $\mathrm{GeH}_{4}$ flow was $629 \mu \mathrm{mol} / \mathrm{min}$. The incubation times and exact growth rates of subsequent Ge layers were again difficult to determine from the reflectivity results.

A significant density of stacking faults was observed in all samples grown at $500^{\circ} \mathrm{C}$. Stacking faults were also observed in $650^{\circ} \mathrm{C}$ samples grown at 100Torr, but not in the samples grown at 250Torr.

\section{Discussion}

\subsection{Ge Morphology on GaAs}


A comparison between the samples grown at 100 Torr (Figs. 1 and 4) indicates a large effect of temperature on the length scale of the Ge surface morphology. This is most clearly observed in the first $\mathrm{Ge}$ layer; at $500^{\circ} \mathrm{C}$ the surface is reasonably planar while at $650^{\circ} \mathrm{C}$ the surface is rough. Wang et al. observed similar results when growing Ge on GaAs using ultrahigh vacuum thermal evaporation [12]. They suggested that thermodynamically, Ge grows on top of GaAs in the Stranski-Krastanov (SK) mode. By growing at a lower temperature, however, the Ge surface diffusion is reduced, which suppresses the Ge clustering that would lead to a rough surface formation [12]. Such a high-level explanation avoids the details of the GaAs surface, which can also be important in affecting the thermodynamic (energy) state. As mentioned previously, Bai et al. determined that the type of atom at the surface of the GaAs layer at a given temperature affects the resulting morphology [11].

A smooth first Ge layer was obtained at $650^{\circ} \mathrm{C}$ when growth pressure was increased to 250 Torr, as seen in sample $5\left(650^{\circ} \mathrm{C} / 250 \mathrm{Torr} /\right.$ anneal only/exact $)$ and sample $9\left(650^{\circ} \mathrm{C} / 250 \mathrm{Torr} / \mathrm{anneal}\right.$ only/exact). In this case, the observed increase in growth rate inhibits Ge cluster formation by reducing the time surface Ge atoms have to diffuse before new Ge atoms are deposited. Note that the smooth Ge layer was obtained without the use of the TMGa pulse. In fact, the samples using the TMGa pulse had highly non-uniform Ge layers.

Experiments by Pristovsek et al. on As desorption from GaAs surfaces indicate that the Ga-rich $\beta 2(2 \times 4)$ surface reconstruction is obtained by annealing GaAs at $650^{\circ} \mathrm{C}$ for 27 seconds [13] in either $\mathrm{H}_{2}$ or $\mathrm{N}_{2}$ ambient. The morphologies of samples $5\left(650^{\circ} \mathrm{C} / 250\right.$ Torr/anneal only/exact $)$ and $9\left(650^{\circ} \mathrm{C} / 250 \mathrm{Torr} / \mathrm{anneal}\right.$ only/exact) suggest that 20 seconds of annealing is sufficient to drive off enough As from the surface to enable Ge to form a relatively uniform layer. 
As detailed in Section 2, the samples grown using a TMGa pulse had a 10 second anneal in $\mathrm{N}_{2}$ ambient prior to and after the pulse. Since a 20 second anneal was sufficient to obtain a uniform Ge layer, the coverage of Ga atoms provided from the TMGa pulse was likely excessive. This result contrasts with those found by Bai et al. and is likely related to the difference in growth temperature. They obtained smooth Ge on GaAs using a TMGa pulse when growing at temperatures below $500^{\circ} \mathrm{C}$, where TMGa partially decomposes to form methylgallium complexes [11]. At $650^{\circ} \mathrm{C}$, however, TMGa is expected to completely decompose and deposit Ga onto the surface. Unlike methylgallium complexes, which form self-limiting monolayers on the GaAs surface, excess Ga atoms are not self-limiting and can build up. It is likely that in the samples using the TMGa pulse, this excess Ga locally inhibited or slowed Ge growth.

\subsection{GaAs Morphology on Ge}

GaAs grown on $\mathrm{Ge}$ at $500^{\circ} \mathrm{C}$ is highly non-uniform; in particular, the morphologies of GaAs in samples $11\left(500^{\circ} \mathrm{C} / 100 \mathrm{Torr} / \mathrm{Ga}\right.$ pulse/exact $)$ and $12\left(500^{\circ} \mathrm{C} / 100 \mathrm{Torr} / \mathrm{Ga}\right.$ pulse/ $6^{\circ}$ off $)$ appear to be islands in various degrees of coalescence. Experiments by Larsen et al. suggest that at low temperatures the reaction to form GaAs occurs only with $\mathrm{TMGa}$ and $\mathrm{AsH}_{3}$ molecules that have been adsorbed onto the surface [14]. It is possible that at $500^{\circ} \mathrm{C}$ the adsorption of $\mathrm{TMGa}$ or $\mathrm{AsH}_{3}$ onto the Ge surface is poor, resulting in slow nucleation of GaAs. With GaAs islands nucleating more slowly, the time and minimum film thickness necessary to achieve a planar layer would be higher. Attempts to grow thin GaAs layers would result in the incomplete states of coalescence and valleys as observed.

In samples grown on (100) $6^{\circ}$ offcut substrates at $650^{\circ} \mathrm{C}$ and 250 Torr (samples 4,6 , 8, and 10), there is a noticeable undulating morphology throughout the film beginning at the GaAs substrate. 
Step-bunching is one possible explanation for the wavy surface of the substrate; many groups have observed step-bunching of grown GaAs on offcut substrates under various conditions, although there is not a clear consensus on some details of the underlying mechanisms [15-17]. The other possible explanation would be the deposition of Ge inducing the wavy surface through an exchange or intermixing mechanism. This is less likely, however, as such a mechanism should reasonably be expected to occur in sample $2\left(650^{\circ} \mathrm{C} / 100 \mathrm{Torr} / \mathrm{Ga}\right.$ pulse $/ 6^{\circ}$ off $)$ as well, with more pronounced undulations due to the slower Ge growth rate.

In samples 5 and $9\left(650^{\circ} \mathrm{C} / 250\right.$ Torr/anneal only/exact $)$, loss of planarity in layers beyond the first Ge layer appears to be caused by APBs in the GaAs layers. This suggests that APBs must be suppressed in order to achieve highly planar GaAs/Ge superlattice layers. Further investigations will be required to achieve this, however, as the results indicate that the traditional method of using offcut substrates to suppress APBs is not sufficient.

\section{Conclusion}

The growth and morphology of GaAs/Ge superlattices grown by MOCVD were investigated for a range of conditions. At $650^{\circ} \mathrm{C}$ and 100 Torr, the surfaces of Ge deposited on GaAs are observed to be rough. This roughening is suppressed when growths are conducted at lower temperatures or higher pressures. This behavior suggests that Ge roughening is a thermodynamically favorable but relatively slow process. At $500^{\circ} \mathrm{C}, \mathrm{GaAs}$ layers grown on $\mathrm{Ge}$ appear as islands of GaAs in incomplete states of coalescence. Poor surface coverage with GaAs is the likely culprit; longer deposition times would be needed to allow for improved coverage or sufficient coarsening of existing GaAs islands to result in a complete film. At $650^{\circ} \mathrm{C}$, antiphase 
boundaries are partly responsible for the non-uniformity in the deposited layers. The use of offcut substrates did not suppress APBs, likely due to the unique conditions involved in the growth of GaAs/Ge superlattices. The range of growth parameters herein allows for the formation of 2-D-like or 3-D-like structures to be investigated for their thermoelectric properties. Although morphologies have been connected to growth parameters in this study, the electronic nature of these interfaces will be important as well, and thus varying the morphology with growth parameters may impact interface electronic structure as well as the morphology described in this report.

\section{Acknowledgements}

This work was supported as part of the Solid State Solar Thermal Energy Conversion Center ( $\left.\mathrm{S}^{3} \mathrm{TEC}\right)$, an Energy Frontier Research Center funded by the U.S. Department of Energy, Office of Science, Office of Basic Energy Sciences under award DE-FG02-09ER46577. This work was also supported under a research grant from the Singapore-MIT Alliance for Research and Technology Low Energy Electronic Systems Program, a research program funded by the National Research Foundation of Singapore. This work made use of the MRSEC Shared Experimental Facilities at MIT, supported by the National Science Foundation under award DMR-14-19807. 


\section{References}

[1] G. Chen, C.L. Tien, X. Wu, J.S. Smith, Thermal Diffusivity Measurement of GaAs/AlGaAs Thin-Film Structures, J. Heat Transf. 116 (1994) 325-331. doi:10.1115/1.2911404.

[2] T. Yao, Thermal properties of AlAs/GaAs superlattices, Appl. Phys. Lett. 51 (1987) 17981800. doi:10.1063/1.98526.

[3] S.-M. Lee, D.G. Cahill, R. Venkatasubramanian, Thermal conductivity of Si-Ge superlattices, Appl. Phys. Lett. 70 (1997) 2957-2959. doi:10.1063/1.118755.

[4] G. Chen, Size and Interface Effects on Thermal Conductivity of Superlattices and Periodic Thin-Film Structures, J. Heat Transf. 119 (1997) 220-229. doi:10.1115/1.2824212.

[5] G. Chen, M. Neagu, Thermal conductivity and heat transfer in superlattices, Appl. Phys. Lett. 71 (1997) 2761-2763. doi:10.1063/1.120126.

[6] R. Dettori, C. Melis, L. Colombo, SixGe1-x alloy as efficient phonon barrier in Ge/Si superlattices for thermoelectric applications, Eur. Phys. J. B. 88 (2015) 1-7. doi:10.1140/epjb/e2014-50628-8.

[7] J. Zhang, N.G. Anderson, K.M. Lau, AlGaAs superlattice microcoolers, Appl. Phys. Lett. 83 (2003) 374-376. doi:10.1063/1.1591242.

[8] P.M. Petroff, A.C. Gossard, A. Savage, W. Wiegmann, Molecular beam epitaxy of Ge and Ga1-xAlxAs ultra thin film superlattices, J. Cryst. Growth. 46 (1979) 172-178. doi:10.1016/0022-0248(79)90053-8.

[9] C.-A. Chang, A. Segmüller, L.L. Chang, L. Esaki, Ge - GaAs superlattices by molecular beam epitaxy, Appl. Phys. Lett. 38 (1981) 912-914. doi:10.1063/1.92179.

[10] T.S. Kuan, C.-A. Chang, Electron microscope studies of a Ge-GaAs superlattice grown by molecular beam epitaxy, J. Appl. Phys. 54 (1983) 4408-4413. doi:10.1063/1.332688.

[11] Y. Bai, K.E. Lee, C. Cheng, M.L. Lee, E.A. Fitzgerald, Growth of highly tensile-strained Ge on relaxed InxGa1-xAs by metal-organic chemical vapor deposition, J. Appl. Phys. 104 (2008) 084518. doi:10.1063/1.3005886.

[12] X.-S. Wang, K.W. Self, W.H. Weinberg, Growth mode of Ge on GaAs(100), J. Vac. Sci. Technol. A. 12 (1994) 1920-1923. doi:10.1116/1.578983.

[13] M. Pristovsek, T. Trepk, M. Klein, J.-T. Zettler, W. Richter, Dynamic study of the surfaces of (001) gallium arsenide in metal-organic vapor-phase epitaxy during arsenic desorption, J. Appl. Phys. 87 (2000) 1245-1250. doi:10.1063/1.372003.

[14] C.A. Larsen, S.H. Li, N.I. Buchan, G.B. Stringfellow, D.W. Brown, Kinetics of the reaction between trimethylgallium and arsine, J. Cryst. Growth. 102 (1990) 126-136. doi:10.1016/0022-0248(90)90893-P.

[15] J. Ishizaki, K. Ohkuri, T. Fukui, Simulation and Observation of the Step Bunching Process Grown on GaAs (001) Vicinal Surface by Metalorganic Vapor Phase Epitaxy, Jpn. J. Appl. Phys. 35 (1996) 1280. doi:10.1143/JJAP.35.1280.

[16] M. Kasu, N. Kobayashi, Surface - diffusion and step - bunching mechanisms of metalorganic vapor - phase epitaxy studied by high - vacuum scanning tunneling microscopy, J. Appl. Phys. 78 (1995) 3026-3035. doi:10.1063/1.360053.

[17] T. Fukui, H. Saito, Natural Superstep Formed on GaAs Vicinal Surface by Metalorganic Chemical Vapor Deposition, Jpn. J. Appl. Phys. 29 (1990) L483. doi:10.1143/JJAP.29.L483. 


\begin{tabular}{|c|c|c|c|c|c|c|}
\hline Sample & $\begin{array}{l}\text { Observed } \\
\text { Superlattice } \\
\text { Period }\end{array}$ & $\begin{array}{c}\text { Substrate } \\
\text { Orientation }\end{array}$ & $\begin{array}{c}\text { Growth } \\
\text { Temperature } \\
\left({ }^{\circ} \mathrm{C}\right)\end{array}$ & $\begin{array}{c}\text { Reactor } \\
\text { Pressure } \\
\text { (Torr) }\end{array}$ & TMGa pulse & $\begin{array}{c}\text { Growth Rate } \\
(\AA / \mathrm{s})\end{array}$ \\
\hline 1 & $\begin{array}{c}55 \mathrm{~nm} \\
\mathrm{GaAs} / 40 \mathrm{~nm} \mathrm{Ge}\end{array}$ & exact (100) & 650 & 100 & $8 \mathrm{sec}, 13 \mu \mathrm{mol} / \mathrm{min}$ & $\begin{array}{c}\text { GaAs: } 1.0-2.3 \\
\text { Ge: } 0-4.1\end{array}$ \\
\hline 2 & $\begin{array}{c}55 \mathrm{~nm} \\
\mathrm{GaAs} / 40 \mathrm{~nm} \mathrm{Ge}\end{array}$ & $\begin{array}{l}(100) 6^{\circ} \text { off } \\
\text { toward }\langle 111>\mathrm{A}\end{array}$ & 650 & 100 & $8 \mathrm{sec}, 13 \mu \mathrm{mol} / \mathrm{min}$ & $\begin{array}{c}\text { GaAs: } 0-2.2 \\
\text { Ge: } 0-4.5\end{array}$ \\
\hline 3 & $\begin{array}{c}\text { 30nm } \\
\mathrm{GaAs} / 70 \mathrm{~nm} \mathrm{Ge}\end{array}$ & exact (100) & 650 & 250 & $5 \mathrm{sec}, 13 \mu \mathrm{mol} / \mathrm{min}$ & $\begin{array}{c}\text { GaAs: } 0.5-2.4 \\
\text { Ge: } 1.4-18.0\end{array}$ \\
\hline 4 & $\begin{array}{c}40 \mathrm{~nm} \\
\mathrm{GaAs} / 60 \mathrm{~nm} \text { Ge }\end{array}$ & $\begin{array}{l}(100) 6^{\circ} \text { off } \\
\text { toward }<111>A\end{array}$ & 650 & 250 & $5 \mathrm{sec}, 13 \mu \mathrm{mol} / \mathrm{min}$ & $\begin{array}{c}\text { GaAs: } 0.4-2.0 \\
\text { Ge: } 2.0-14.0\end{array}$ \\
\hline 5 & $\begin{array}{c}\text { 60nm } \\
\mathrm{GaAs} / 60 \mathrm{~nm} \text { Ge }\end{array}$ & exact (100) & 650 & 250 & No pulse & $\begin{array}{l}\text { GaAs: } 1.0-1.4 \\
\text { Ge: } 10.8-14.4\end{array}$ \\
\hline 6 & $\begin{array}{c}\text { 60nm } \\
\text { GaAs/60nm Ge }\end{array}$ & $\begin{array}{c}(100) 6^{\circ} \text { off } \\
\text { toward }<111>A\end{array}$ & 650 & 250 & No pulse & $\begin{array}{l}\text { GaAs: } 1.1-1.4 \\
\text { Ge: } 10.4-14.0\end{array}$ \\
\hline 7 & $\begin{array}{c}20 \mathrm{~nm} \\
\mathrm{GaAs} / 25 \mathrm{~nm} \text { Ge }\end{array}$ & exact (100) & 650 & 250 & $5 \mathrm{sec}, 13 \mu \mathrm{mol} / \mathrm{min}$ & $\begin{array}{c}\text { GaAs: } 0-1.9 \\
\text { Ge: } 0-12.4\end{array}$ \\
\hline 8 & $\begin{array}{c}20 \mathrm{~nm} \\
\mathrm{GaAs} / 25 \mathrm{~nm} \mathrm{Ge}\end{array}$ & $\begin{array}{c}(100) 6^{\circ} \text { off } \\
\text { toward }<111>\mathrm{A}\end{array}$ & 650 & 250 & $5 \mathrm{sec}, 13 \mu \mathrm{mol} / \mathrm{min}$ & $\begin{array}{l}\text { GaAs: } 0-1.7 \\
\text { Ge: } 0-12.8\end{array}$ \\
\hline 9 & $\begin{array}{c}\text { 30nm } \\
\mathrm{GaAs} / 30 \mathrm{~nm} \mathrm{Ge}\end{array}$ & exact (100) & 650 & 250 & No pulse & $\begin{array}{l}\text { GaAs: } 1.0-1.6 \\
\text { Ge: } 10.0-14.0\end{array}$ \\
\hline 10 & $\begin{array}{c}30 \mathrm{~nm} \\
\mathrm{GaAs} / 30 \mathrm{~nm} \text { Ge }\end{array}$ & $\begin{array}{c}(100) 6^{\circ} \text { off } \\
\text { toward }<111>\mathrm{A}\end{array}$ & 650 & 250 & No pulse & $\begin{array}{c}\text { GaAs: } 0-2.6 \\
\text { Ge: } 10.0-12.8\end{array}$ \\
\hline 11 & $\begin{array}{c}\text { 50nm } \\
\text { GaAs/10nm Ge }\end{array}$ & exact (100) & 500 & 100 & $\begin{array}{l}15 \mathrm{sec}, 34 \\
\mu \mathrm{mol} / \mathrm{min}\end{array}$ & GaAs: 0-3.6 \\
\hline 12 & $\begin{array}{c}50 \mathrm{~nm} \\
\mathrm{GaAs} / 10 \mathrm{~nm} \mathrm{Ge}\end{array}$ & $\begin{array}{c}(100) 6^{\circ} \text { off } \\
\text { toward }<111>\mathrm{A}\end{array}$ & 500 & 100 & $\begin{array}{l}15 \mathrm{sec}, 34 \\
\mu \mathrm{mol} / \mathrm{min}\end{array}$ & GaAs: $0-3.1$ \\
\hline 13 & $\begin{array}{c}55 \mathrm{~nm} \\
\mathrm{GaAs} / 20 \mathrm{~nm} \text { Ge }\end{array}$ & exact (100) & 500 & 250 & $5 \mathrm{sec}, 34 \mu \mathrm{mol} / \mathrm{min}$ & GaAs: $1.0-3.7$ \\
\hline 14 & $\begin{array}{c}55 \mathrm{~nm} \\
\mathrm{GaAs} / 20 \mathrm{~nm} \text { Ge }\end{array}$ & $\begin{array}{c}(100) 6^{\circ} \text { off } \\
\text { toward }\langle 111>\mathrm{A}\end{array}$ & 500 & 250 & $5 \mathrm{sec}, 34 \mu \mathrm{mol} / \mathrm{min}$ & GaAs: $1.6-3.2$ \\
\hline
\end{tabular}

Table 1: Summary of grown structures and epitaxy conditions. Thicknesses of the GaAs and Ge layers were determined using TEM. For samples with non-uniform layers, approximate average thicknesses are given. For samples 11 -14, thicknesses given do not reflect the thickness of the first Ge layer deposited; Ge growth rates are not determined due to difficulty in separating out incubation time. 

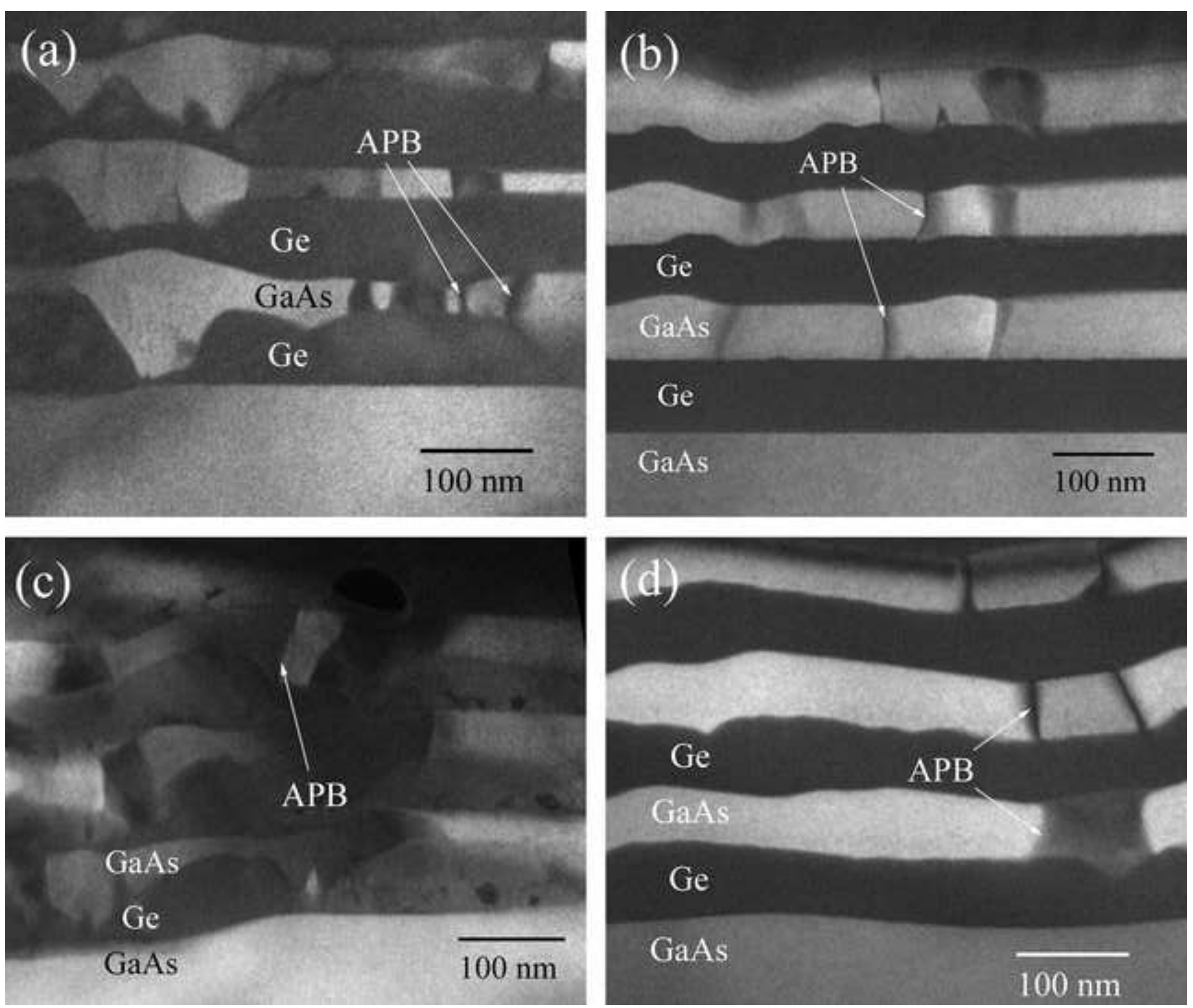


\section{(a)}

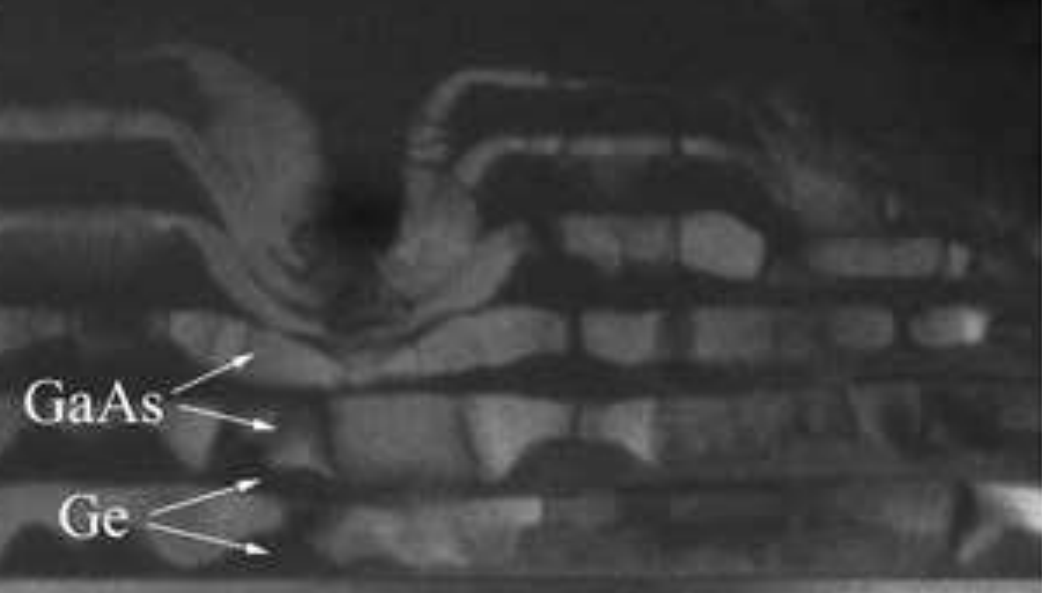

GaAs
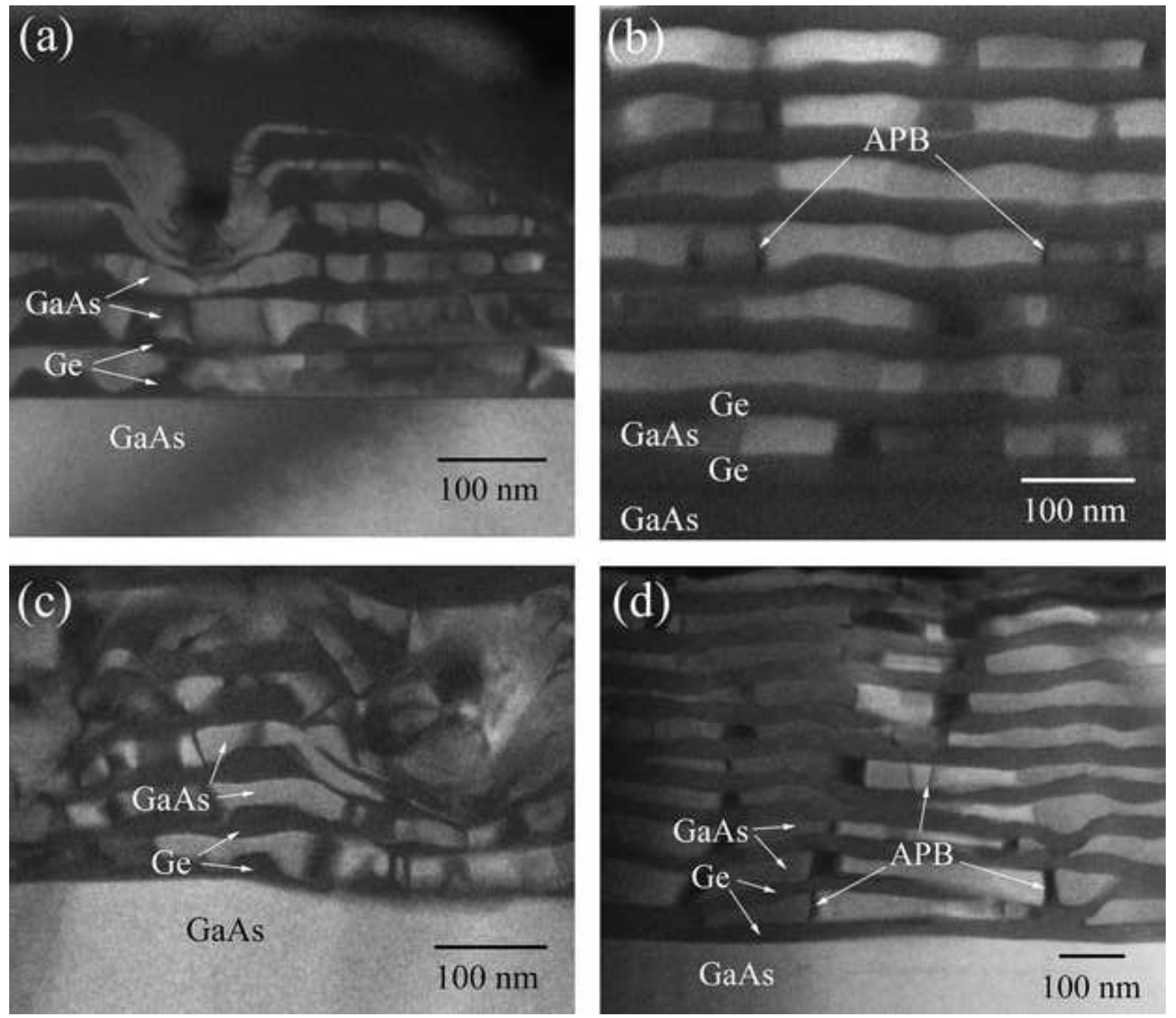


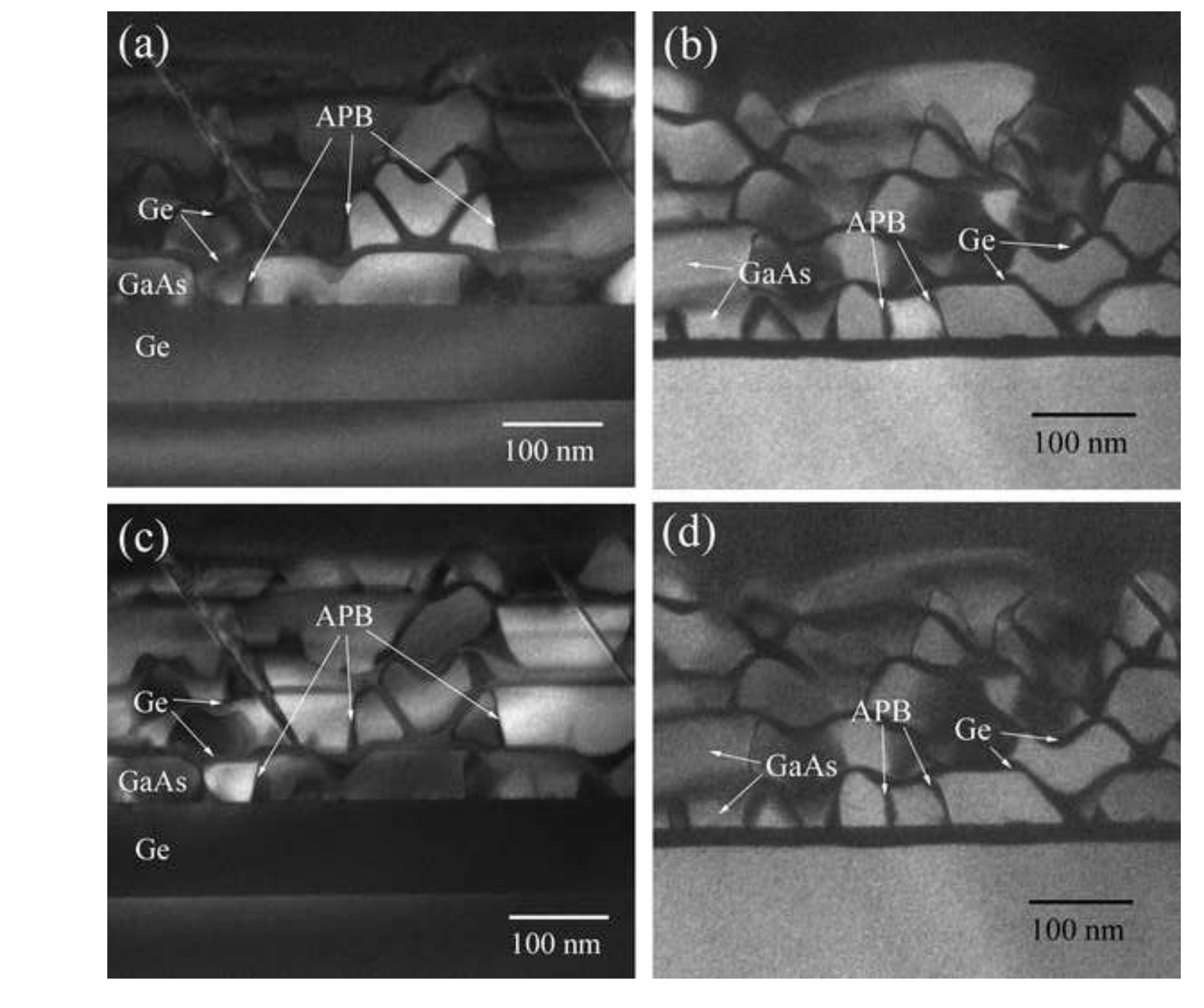



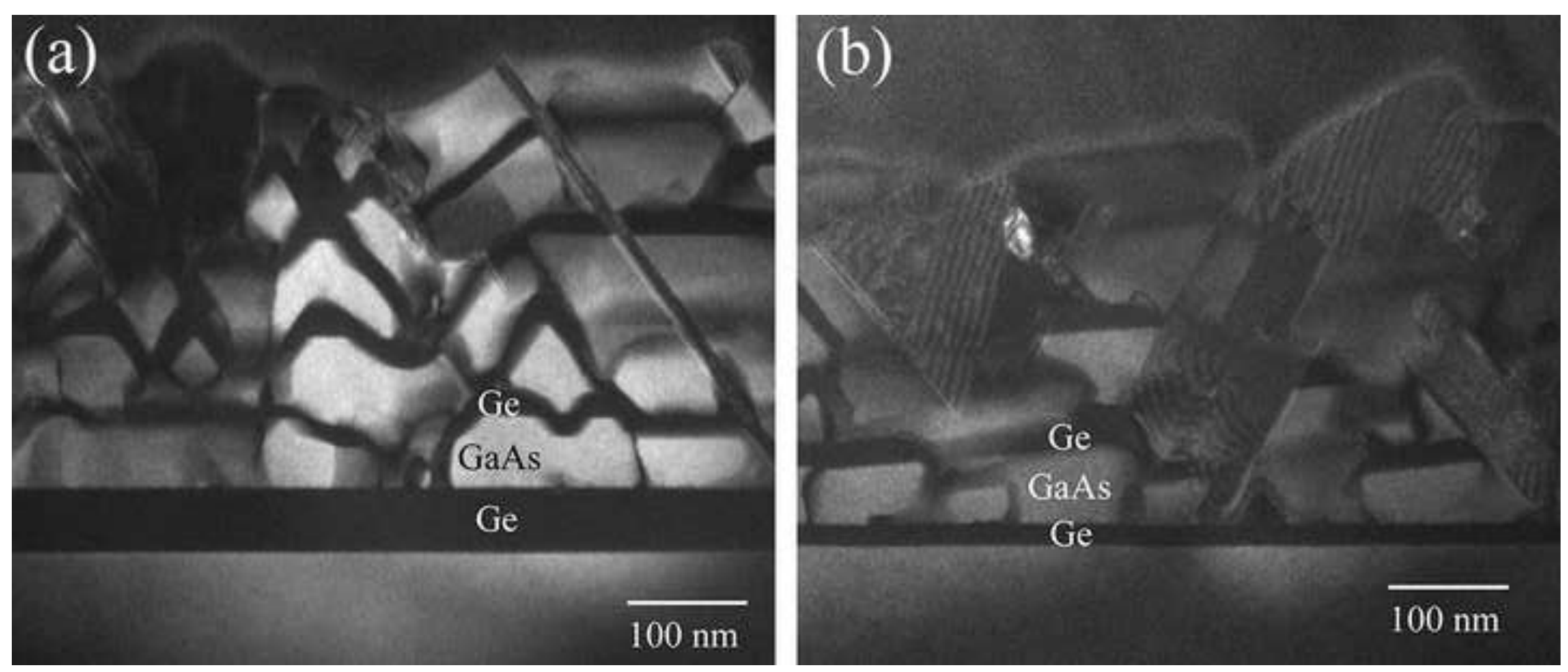

(b) GaAs Ge 